\title{
Functional Neurorehabilitation using the Hybrid Assistive Limb (HAL): A First Experience in the United States
}

Emre Yilmaz*, Christian Fisahn, Angeli Mayadev, Kim Kobota, Ziadee Cambier, Cameron K Schmidt, Daniel C Norvell and Jens Chapman

Swedish Neuroscience Institute, Swedish Medical Center, Seattle, Washington, USA

\begin{abstract}
Introduction: The Hybrid Assistive Limb (HAL, Cyberdyne, Japan) facilitates voluntary, user-driven ambulation through a neurologically-controlled system based on bioelectrical signals derived from the user. This allows for the repeated execution of physiologically faithful gait patterns, crucial to recovery in cases of neurologic motor deficit. In this series, we present the first three patients in the United States to undergo HAL neurorehabilitation training.
\end{abstract}

Patient and methods: A case series of three patients participating in a single-center prospective, interventional pilot study, suffering neurologic motor deficits secondary to spinal cord infarct following a pulmonary embolism (patient 1), multiple sclerosis (patient 2) and the surgical resection of a petroclival meningioma (patient 3). The patients underwent 60 sessions of body weight-supported treadmill training in the HAL over the course of 12 weeks. Measures of functional ambulation (10 Minute Walk Test, 10MWT) were performed out of the HAL before and after each session and at the 12 week and 6 month follow-up. Timed Up \& Go (TUG) test was performed each week. Treadmill data (time, distance) while in HAL was recorded at each session. Measures of endurance (6 Minute Walk Test, 6MWT), risk of fall (TUG), balance impairment (Berg Balance Scale) and improvements in walking performance (Walking Index for Spinal Cord Injury II, WISCI II) were measured at baseline, after 12 weeks and at 6 months follow-up.

Results: Patients 2 and 3 completed 60 visits, patient 1 completed 56 visits. All patients achieved markedly increased treadmill paces, improved functional scores, increased distance in the 6MWT and decreased TUG times at 6 -month follow-up. In the 10MWT, all patients achieved a clinically significant decrease in time and steps and showed improvements in the required assistance level to perform the test. Patients 1 and 3 showed improvement on the Berg Balance Scale. Patient 2 had no change between baseline and 6-month follow-up. Only minor adverse effects were reported, including skin abrasions and irritation secondary to chaffing of the HAL unit and EMG electrodes.

Conclusion: These data show that HAL training is both feasible and effective in the neurorehabilitation of patients suffering neurologic motor deficits secondary to trauma and/or pathological/neurodegenerative processes after they have undergone normal rehab. A greater number of patients are required to meaningfully assess the differences in improvement from baseline, based upon underlying pathologies.

Keywords: Hybrid assistive limb; HAL; Exoskeleton; Neurorehabilitation; Gait training; Treadmill training

\section{Introduction}

The treatment of neurologic motor deficits following spinal cord injury (SCI), stroke (CVA), multiple sclerosis (MS) and other neuromuscular disorders continues to pose a major challenge for the affected patients, their families and health care systems as a whole due to the absence of meaningful restorative therapies. To date, there has been no major breakthrough therapeutic success beyond trying to gain the best possible functional independence given the respective impairments of these patients.

Several neurorehabilitation therapies exist for patients suffering from neurologic motor dysfunction ranging from conventional gait training, balance and strength training, neuromuscular electrical stimulation, treadmill training and drug therapy. The generally accepted timeframe for plateauing after functional interventions is three months [1-4].

More recently, exoskeletons such as Re-Walk (Argo Medical Technologies Ltd, Yokneam Ilit, Israel), Rex-Bionics (Auckland, New Zealand) or Wearable Power-Assist Locomotor exoskeleton (WPAL; Fujita Health University, Japan) have been introduced to improve mobility. The principle of an exoskeletal support as well as functional myoelectric stimulation for neurorehabilitation has been used for many years [5-9]. In comparison to these passive exoskeletons, the concept of the interactive neurologically-controlled exoskeleton Hybrid Assistive Limb (HAL', Cyberdyne Inc. and Japan) combines both principles. $\mathrm{HAL}^{\circ}$ uses a neurologically-controlled system to detect minute surface action potentials on the patient's skin surface and assists user-driven locomotive action based on these signals, allowing patient's a voluntarily driven range of motion [10].

Several international studies have demonstrated the safety and feasibility of the HAL in the context of several conditions, including CVA, SCI, both chronic incomplete SCI and chronic complete SCI with zones of partial preservation (ZPP) and in other cases of neurologic motor dysfunction secondary to traumatic events and/or neurodegenerative pathologies. Furthermore, these studies have shown that training with HAL improves quality of life, decreases neuropathic pain and increases patient's functional mobility, over-ground walking, muscle strength and motor function in and out of $\mathrm{HAL}^{\circ}$ [11-14].

\section{Purpose of the Study}

In this series, we present the first three patients in the United States to undergo $\mathrm{HAL}^{\circ}$ neurorehabilitation training.

*Corresponding author: Emre Yilmaz, MD, Swedish Neuroscience Institute, Swedish Medical Center, 550 17th Avenue, Suite 500 James Tower, 5th Floor, Seattle, WA 98122, United States, Tel: (206) 399-1438; E-mail: cavallil.doc@gmail.com

Received February 08, 2018; Accepted February 19, 2018; Published February 27, 2018

Citation: Yilmaz E, Fisahn C, Mayadev A, Kobota K, Cambier Z, et al. (2018) Functional Neurorehabilitation using the Hybrid Assistive Limb (HAL): A Firs Experience in the United States. Int J Neurorehabilitation 5: 306. doi: 10.4172/23760281.1000306

Copyright: @ 2018 Yilmaz E, et al. This is an open-access article distributed under the terms of the Creative Commons Attribution License, which permits unrestricted use, distribution, and reproduction in any medium, provided the original author and source are credited. 


\section{Patients and Methods}

We present the first three patients participating in a single-center prospective, interventional pilot study, who were suffering from chronic neurologic motor deficits secondary to spinal cord infarct following a pulmonary embolism (patient 1), MS (patient 2) and the surgical resection of a petroclival meningioma (patient 3). Patients were included who had achieved a stable non-progressive state in their motor neurologic deficit following (a) SCI with American Spinal Injury Association (ASIA) A through D functional status with thoracic and lumbar levels of paralysis (b) cervical spinal cord injury with incomplete injuries below C6, or (c) stroke, MS, or other neurodegenerative disorders that cause significant gait impairment. Patients on active medications for spasticity were required to be on a stable dose for at least three months prior to study entry.

\section{Patient 1}

31 year old female with a 6 week old spinal cord infarction after cardiac arrest during surgery resulting in a T12 ASIA C paraplegia.

\section{Patient 2}

60 year old male with a 20 year history of relapsing-remitting MS. He has always been on disease modifying therapy for MS and is currently on Fingolimod. His MS expanded disability status scale (EDS) score prior to the study was 6.5 , which remained constant throughout the study.

\section{Patient 3}

54 year old female with initially left greater than right hemiparesia and significant ongoing bilateral ataxia status following resection of a large right petroclivial meningioma resection 6 months ago.

All patients underwent 60 sessions of Body Weight-Supported Treadmill Training (BWSTT) in the HAL over the course of 12 weeks (Figures 1 and 2). All training was performed under the supervision of a trained neuro physiotherapist in the Swedish Multiple Sclerosis Center rehabilitation facility. Required level of assistance, number of steps and time were assessed using the 10 Minute Walk Test (10MWT) out of the HAL' before and after each session [15]. The 10MWT was also evaluated at baseline, 6 weeks and at the 12 week and 6 month
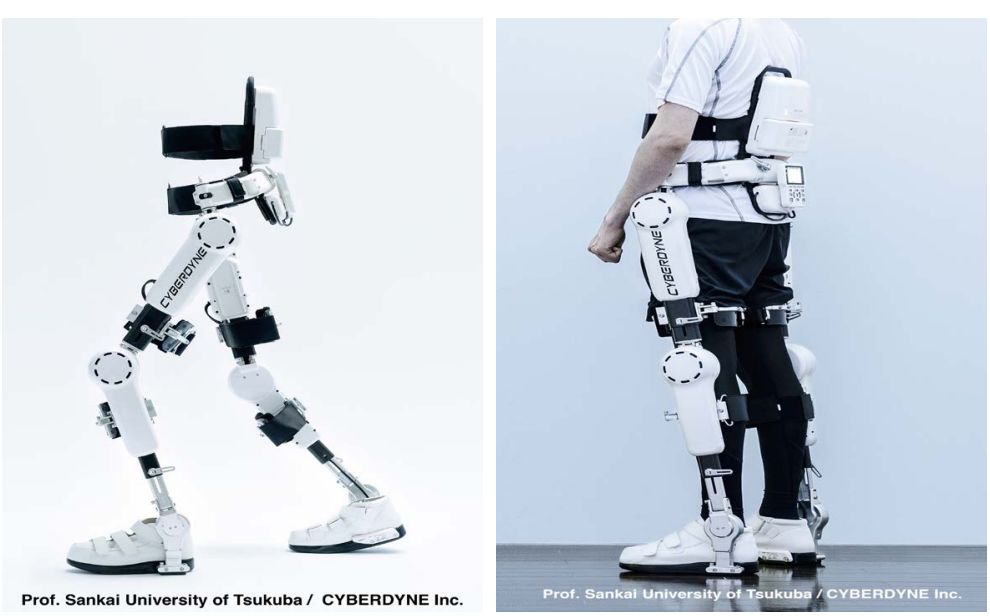

Note: Pictures of a HAL ${ }^{\circledR}$ exoskeleton (left/right). With written consent obtained from CYBERDYNE Inc. Copyright and courtesy of CYBERDYNE Inc. Figure 1: The $\mathrm{HAL}^{\circledR}$ exoskeleton.

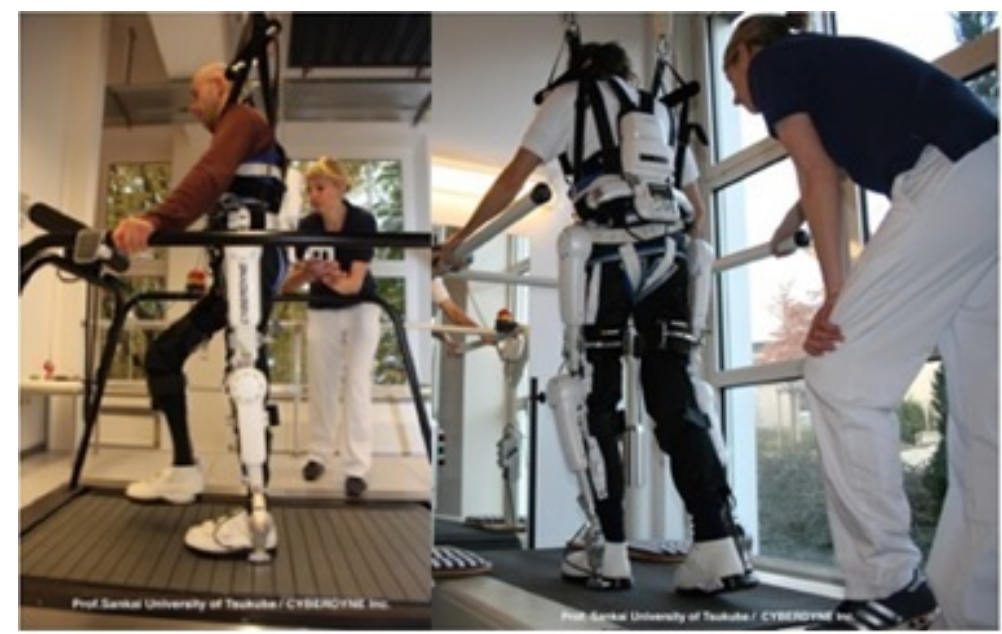

Figure 2: Training on a treadmill with $\mathrm{HAL}^{\circledR}$

All patients underwent 60 sessions of Body Weight Supported Treadmill Training (BWSTT) in the HAL ${ }^{\circledR}$. All taring was performed under the supervision of a trained neuro physiotherapist. With written consent obtained from CYBERDYNE Inc. Copyright and courtesy of CYBERDYNE Inc. 
follow-up. The Timed Up \& Go (TUG) test was performed each week, measuring the time and assistance needed for standing up from a chair, walking $3 \mathrm{~m}$, turning around, walking back and sitting down. Treadmill data (time, distance, blood pressure, heart rate) while in $\mathrm{HAL}^{\circ}$ was recorded at each session. Measures of endurance (6 Minute Walk Test, 6MWT), risk of fall (TUG), balance impairment (Berg Balance Scale) and improvements in walking performance (WISCI II) were measured at baseline, 12 weeks training and 6 month follow-up. The WISCI II score is a 20 item scale measuring the walking capabilities of a patient based on the requirements of assistance from walking aids, personal assistance or braces. Grade 0 means that the patient has neither standing nor walking abilities. Grade 20 means that no assistance is needed to walk a distance of $10 \mathrm{~m}$ [16-19].

\section{Results}

All three patients with an age of 31,60 and 54 underwent protocolled treadmill training with $\mathrm{HAL}^{\circ}$ in 2016. Patient 1 completed 56 out of 60 visits. Patients 2 and 3 completed 60 visits. The mean WISCI II score increased from 5.0 at baseline, to 11.3 at the 6-month follow-up (Figure 3). Improvements in speed and endurance were achieved in all patients (Tables 1 and 2). The required time for the 10MWT decreased on average by $21.1 \%$ and the mean number of steps decreased by $17.0 \%$ (Table 1). The mean time to complete the TUG test decreased by $11.3 \%$ and the mean walk distance covering during the $6 \mathrm{MWT}$ improved by $21.1 \%$ (Figures 4 and 5). The results are summarized in Tables 1 and 2.

\section{Patient 1}

The WISCI II score improved from 1 at baseline to 13 at the 6 month follow-up (Figure 3). The time to complete the 10MWT at 6 months follow-up was reduced by $11.7 \mathrm{~s}$ relative to baseline $(64.7 \mathrm{~s}$ vs. $53.0 \mathrm{~s}$ ). The number of steps needed to complete the 10MWT at 6 months follow-up decreased by 8 steps compared to baseline (42 steps

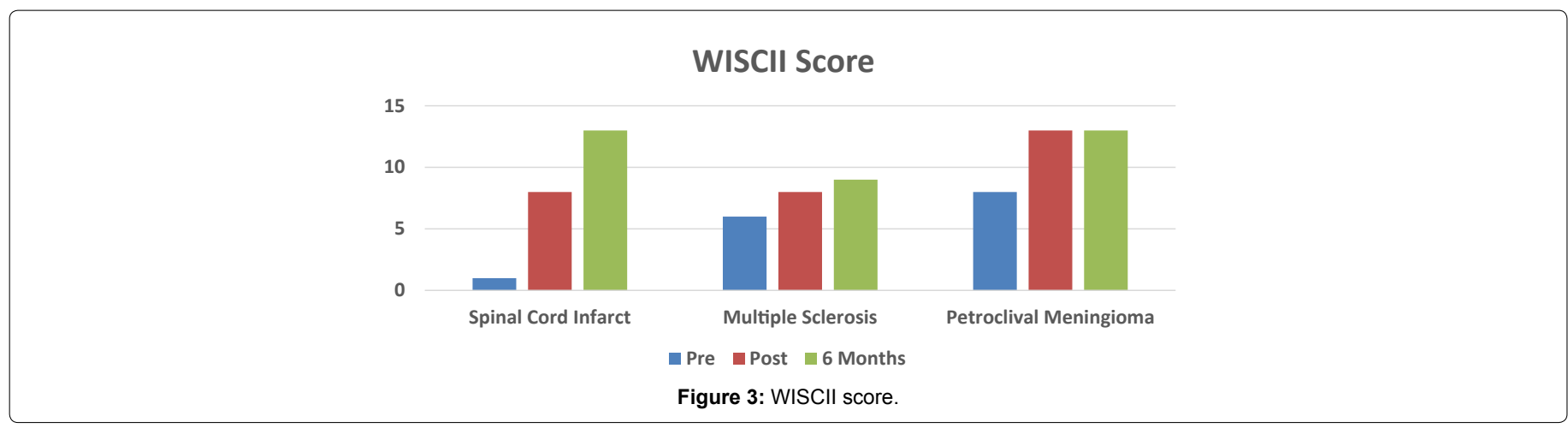

\begin{tabular}{|c|c|c|c|c|c|c|c|c|}
\hline Spinal Cord Infarct & Baseline & Tx start & 6 week & Last day & 12 weeks & 6 months & Change* $^{*}$ & Change $\%$ \\
\hline VISIT & 0 & 1 & 30 & 60 & & 62 & & \\
\hline WISCII & 1 & - & - & - & 8 & 13 & 12 & \\
\hline 10 Meter Walk Test (s) & 64.7 & 37.0 & 36.8 & - & 60 & 53 & -11.7 & $-18.1 \%{ }^{*}$ \\
\hline Assistance Level & - & $\min A$ & cga & - & $\bmod a$ & sup & - & \\
\hline 10 Meter Test (steps) & 42 & 24 & 32 & - & 38 & 34 & -8 & $-19.1 \%{ }^{*}$ \\
\hline Tug Time Before & - & - & - & - & 92 & 81 & & $-12.0 \%$ ** \\
\hline 6 Minute Walk Distance & - & - & - & - & 43 & 60 & & $+39.5 \%$ ** \\
\hline BERG & 7 & - & - & - & 9 & 9 & 2 & \\
\hline Multiple Sclerosis & Baseline & Tx start & 6 week & Last day & 12 weeks & 6 months & Change* $^{*}$ & Change \% \\
\hline VISIT & 0 & 1 & 30 & 60 & 61 & 62 & & \\
\hline WISCII & 6 & - & - & - & 8 & - & - & \\
\hline 10 Meter Walk Test (s) & 25.6 & 22.4 & 24.9 & 17.6 & 20.6 & 19.1 & -7.5 & $-25.4 \%^{*}$ \\
\hline Assistance Level & sbg & cga & cga & cga & sup & sup & - & \\
\hline 10 Meter Test (steps) & 24 & 22 & 24 & 19 & 22 & 19 & & $-20.8 \%{ }^{*}$ \\
\hline Tug Time Before & 29.8 & - & 31.2 & - & 30.9 & 27.9 & -1.9 & $-6.4 \% *$ \\
\hline 6 Minute Walk Distance & 104.1 & - & - & - & 104.5 & - & & $+0.4 \% * * *$ \\
\hline BERG & 29 & - & - & - & 28 & - & - & \\
\hline Petroclival Meningioma & Baseline & Tx start & 6 week & Last day & 12 weeks & 6 months & Change* & Change \% \\
\hline VISIT & 0 & 1 & 30 & 60 & 61 & 62 & & \\
\hline WISCII & 8 & - & - & - & 13 & 13 & 5 & \\
\hline 10 Meter Walk Test (s) & 42.7 & 40.0 & 47.1 & 34.6 & 33.8 & 34.1 & -8.6 & $-20.1 \%{ }^{*}$ \\
\hline Assistance Level & cga & cga & sbg & sbg & sbg & $\operatorname{sbg}$ & - & \\
\hline 10 Meter Test (steps) & 27 & 26 & 28 & 25 & 26 & 24 & -3 & $-11.1 \% *$ \\
\hline Tug Time Before & 80.8 & - & - & - & 53.6 & 65.9 & -14.9 & $-18.4 \%{ }^{*}$ \\
\hline 6 Minute Walk Distance & 66.2 & - & - & - & 87.3 & 81.6 & 15.5 & $+23.3 \% *$ \\
\hline BERG & 13 & - & - & - & 21 & 25 & 12 & \\
\hline
\end{tabular}

Change*: Difference from baseline; ${ }^{* *}$ : Change 12 weeks to 6 months; ${ }^{* * *}$ : Change 12 weeks to baseline; - Blank cells represent missing data, Tx: Training Start ${ }_{\text {min }}$; a: Minimum Assistence; cga: Contact Guard; ma: Moderate Assisstence; sup: Suplement; sbg: Standy-By Guard

Table 1: Shows the changes of walking abilities during the time of treadmill training and the 6 months follow-up for all three patients 
vs. 34 steps). The assistance level improved from, minimum assistance "to „supplemental "at the 6 month follow-up. Relative to the 12 week reevaluation (92 s) the time to complete the TUG Test (Figure 4) decreased by $11 \mathrm{~s}$ at 6 months follow-up (81 s). Relative to the 12-weeks

\begin{tabular}{|c|c|c|c|}
\hline & Start (miles) & Min-max (miles) & End (miles) \\
\hline SCI & 0.02 & $0.02-0.41$ & 0.17 \\
\hline MS & 0.18 & $0.16-0.46$ & 0.36 \\
\hline PM & 0.08 & $0.08-0.6$ & 0.57 \\
\hline
\end{tabular}

SCl: Spinal Cord Infarct; MS: Multiple Sclerosis; PM: Petroclival Meningioma Table 2: Distance covered on a treadmill. follow-up (43 m), distance covered during 6MWT (Figure 5) increased by $17 \mathrm{~m}$ at 6 months follow-up $(60 \mathrm{~m})$. At the 6-month follow-up ( 9 points) the patient's BERG balance (Figure 6) score had increased two points relative to baseline (7 points). Between the first and the last $\mathrm{HAL}^{\circ}$ training session the patient increased the distance covering on the treadmill from 0.02 miles to 0.17 miles (Figure 7). The results are summarized in Tables 1 and 2 .

\section{Patient 2}

The WISCI II score improved from 6 at baseline to 8 at the 3 -month follow-up (Figure 3). The time to complete the 10MWT at 6 months
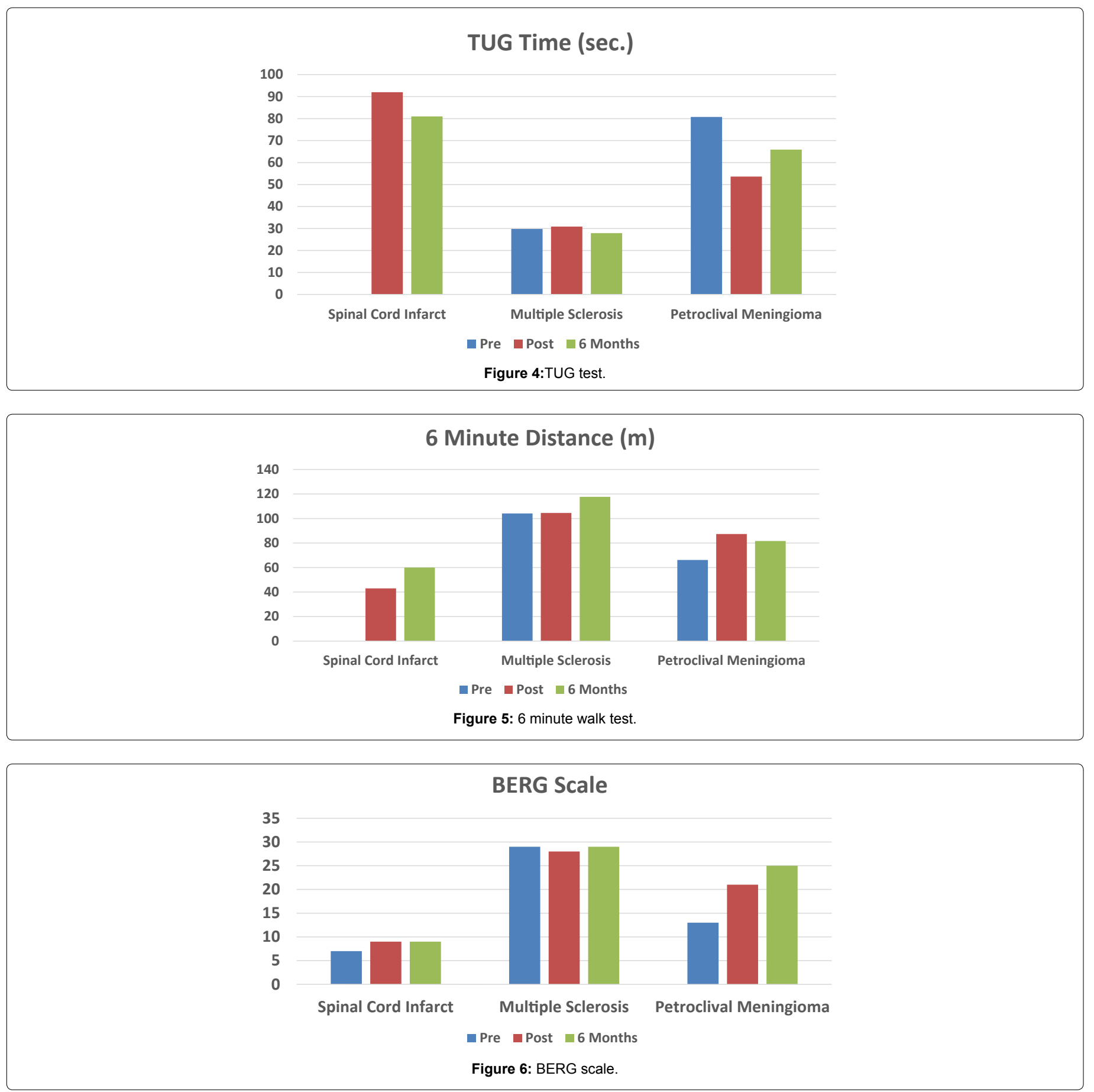


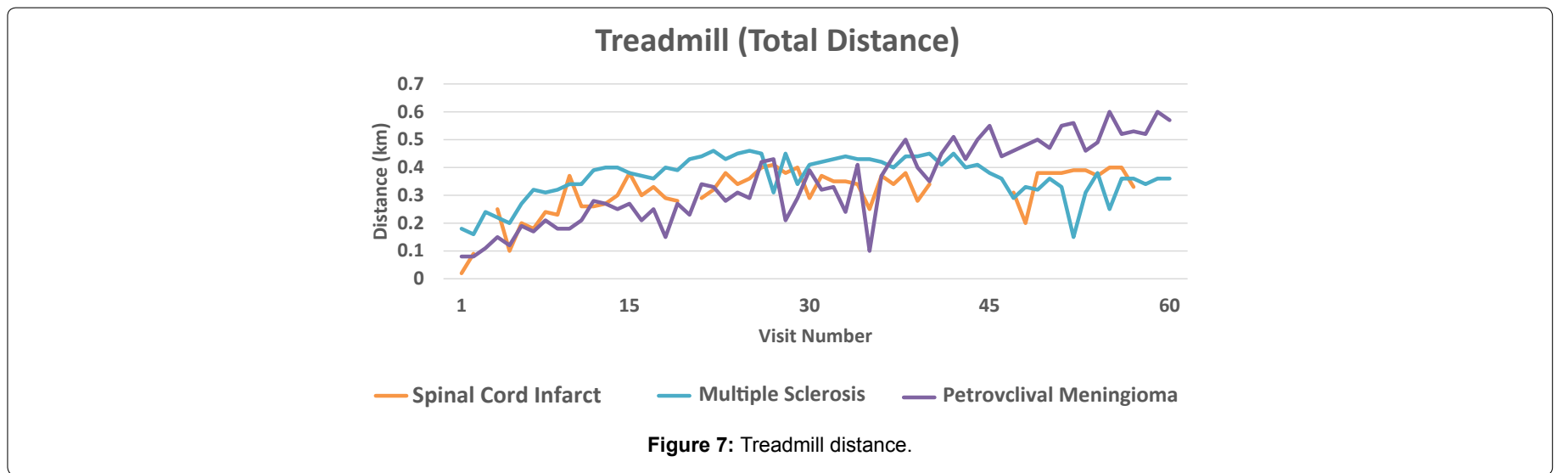

follow-up was reduced by $7.5 \mathrm{~s}$ relative to baseline $(25.6 \mathrm{~s}$ vs. $19.1 \mathrm{~s})$. The number of steps needed to complete the 10MWT at 6-months followup decreased by 5 steps compared to baseline ( 24 steps vs. 19 steps). The assistance level improved from "stand-by guard" to "supplemental" at the 6 month follow-up. Relative to baseline $(29.8 \mathrm{~s})$ the time to complete the TUG Test (Figure 4 ) decreased by $1.9 \mathrm{~s}$ at 6 months follow-up (27.9 s). Relative to baseline $(104.1 \mathrm{~m})$, the distance covered during the 6MWT (Figure 5) increased to $140.5 \mathrm{~m}$ at 3 months follow-up. At the 3 months follow-up (28 points) the patients BERG balance score (Figure 6 ) had decreased one point relative to baseline ( 29 points). The results are summarized in Tables 1 and 2. The ambulated distance on the treadmill at the first session was 0.18 miles and increased to 0.36 miles at the end of the $\mathrm{HAL}^{\circ}$ training (Figure 7). Prior to $\mathrm{HAL}^{\circ}$ training, the patient was unable to stand and was taking fewer steps with his walker in a lurching gait pattern. After the study, the patient was able to stand unsupported for short periods and walks more smoothly with a walker.

\section{Patient 3}

The WISCI II score improved from 8 at baseline to 13 at the 6 month follow-up (Figure 3). The time to complete the $10 \mathrm{MWT}$ at 6 months follow-up was reduced by $8.6 \mathrm{~s}$ relative to baseline ( $42.7 \mathrm{~s}$ vs. $34.1 \mathrm{~s})$. The number of steps needed to complete the 10MWT at 6-months followup decreased by 3 steps compared to baseline ( 27 steps vs. 24 steps). The assistance level improved from "contact guard" to "stand-by guard" at the 6 month follow-up. Relative to the 12 week reevaluation ( 80.8 s) time to complete the TUG Test (Figure 4) decreased by $14.9 \mathrm{~s}$ at 6 months follow-up $(65.9 \mathrm{~s})$. Relative to baseline $(66.2 \mathrm{~m})$, the distance covered during 6MWT (Figure 5) had increased by $15.5 \mathrm{~m}$ at 6 months follow-up $(81.6 \mathrm{~m})$. At the 6-month follow-up (25 points) the patients BERG balance score (Figure 6) had increased 12 points relative to baseline (13 points). The distance covered on the treadmill improved from 0.08 miles to 0.57 miles at the end of treadmill training with $\mathrm{HAL}^{\circ}$ (Figure 7). The results are summarized in Tables 1 and 2).

Only minor adverse effects were reported, including skin abrasions and irritation secondary to chaffing of the HAL unit and EMG electrodes.

\section{Discussion}

This study describes the first cohort in the United States undergoing treadmill training with $\mathrm{HAL}^{\circ}$. The purpose of this study was to determine whether training with $\mathrm{HAL}^{\circ}$ improves functional mobility in patients with neurologic disorders or spinal cord injuries.

Treadmill training with $\mathrm{HAL}^{\circ}$ is postulated to aid cortical plasticity and the restoration of spinal reflex circuits through the recruitment and repeated use of remaining somatosensory afferent pathways and corticospinal tracts $[12,20]$. Patient 3 experienced a decline in performance in the 6MWT and TUG test between the 12 week and 6 month follow-up. This may evidence regression without $\mathrm{HAL}$ training. Furthermore, electrophysiological data has demonstrated a normalization of primary somatosensory cortex activation, the disinhibiting and reorganization of which is a staple of spinal cord injury [12].

Although evidence is only beginning to emerge in the literature, the effectiveness of bodyweight supported treadmill training with HAL in patients with spinal cord injuries and neurologic disorders has been analyzed and suggests promising results [13,21-23].

All patients achieved markedly increased treadmill performance, increased WISCI II scores, increased distance in the 6MWT and decreased TUG Test times at 6-months follow-up. In the 10MWT, all patients achieved a decrease in time and steps and showed improvements in the required assistance level to perform the test. Patients 1 and 3 showed improvement on the Berg Balance Scale. Patient 2 had no change between baseline and the 6-month follow-up. These results imply HAL supported treadmill training can improve walking abilities and motor function in patients with neurologic disorders or spinal cord injuries. Our results in this limited pilot study suggest that treadmill training with $\mathrm{HAL}^{\circ}$-support can improve walking abilities in terms of speed, gait and distance in spinal cord injury and brainstem lesion patients and may have some more limited benefit in stroke patients.

This study has several limitations, including the small number of patients $(n=3)$ and the heterogenous group of patients. However, all patients were treated by the same multidisciplinary team, according to a standrized protocol in the same facility. During this first trail only minor adverse effects were reported, including skin abrasions and irritation secondary to chaffing of the $\mathrm{HAL}^{\circ}$ unit and EMG electrodes.

\section{Conclusion}

These data show that $\mathrm{HAL}^{\bullet}$ training is both feasible and effective in the neurorehabilitation of patients suffering neurologic motor deficits secondary to trauma and/or pathological/neurodegenerative processes after they have undergone normal rehab. A greater number of patients are required to meaningfully assess the functional improvement based upon underlying pathologies. Further studies in the form of randomized trials are needed to assess the comparative effectiveness of the changes in functional mobility and to determine which patients benefit from training with neurologically-controlled exoskeletons. 
Citation: Yilmaz E, Fisahn C, Mayadev A, Kobota K, Cambier Z, et al. (2018) Functional Neurorehabilitation using the Hybrid Assistive Limb (HAL): A First Experience in the United States. Int J Neurorehabilitation 5: 306. doi: 10.4172/2376-0281.1000306

\section{References}

1. Takeda K, Tanino G, Miyasaka H (2017) Review of devices used in neuromuscular electrical stimulation for stroke rehabilitation. Med Devices (Auckl) 10: 207-213.

2. Barbeau H (2003) Locomotor training in neurorehabilitation: Emerging rehabilitation concepts. Neurorehabil Neural Repair 17: 3-11.

3. Behrman AL, Harkema SJ (2000) Locomotor training after human spinal cord injury: A series of case studies. Phys Ther 80: 688-700.

4. Dietz V, Harkema SJ (2004) Locomotor activity in spinal cord-injured persons. J Appl Physiol 96: 1954-1960.

5. Hussey RW, Stauffer ES (1973) Spinal cord injury: Requirements for ambulation. Arch Phys Med Rehabil 54: 544-547.

6. Rosman N, Spira E (1974) Paraplegic use of walking braces: A survey. Arch Phys Med Rehabil 55: 310-314.

7. Summers BN, McClelland MR, Masri el WS (1988) A clinical review of the adult hip guidance orthosis (Para Walker) in traumatic paraplegics. Paraplegia 26: 19-26

8. Vodovnik L, Vodovnik L, Long C, Reswick JB, LippayA, et al. (1965) Myoelectric control of paralyzed muscles. IEEE Trans Biomed Eng 12: 169-72.

9. Vodovnik L, Rebersek S (1974) Information content of myo-control signals for orthotic and prosthetic systems. Arch Phys Med Rehabil 55: 52-56.

10. Kawamoto H, Sankai Y (2002) Power Assist System HAL-3 for gait disorder person. In: ICCHP '02 Proceedings of the 8th International Conference on Computers Helping People with Special Needs.

11. Cruciger O, egenthoff M, Schwenkreis P, Schildhauer TA, Aach M (2014) Locomotion training using voluntary driven exoskeleton (HAL) in acute incomplete SCI. Neurology 83: 474.

12. Sczesny-Kaiser M, Höffken $O$, Aach M, Cruciger O, Grasmücke D, et al. (2015) HAL exoskeleton training improves walking parameters and normalizes cortica excitability in primary somatosensory cortex in spinal cord injury patients. $J$ Neuroeng Rehabil 12: 68.

13. Grasmücke D, Zieriacks A, Jansen $O$, Fisahn $C$, Sczesny-Kaiser $M$, et al (2017) Against the odds: What to expect in rehabilitation of chronic spinal cord injury with a neurologically controlled hybrid assistive limb exoskeleton. A subgroup analysis of 55 patients according to age and lesion level. Neurosurg Focus 42: E15.

14. Aach M, Meindl RC, Geßmann J, Schildhauer TA, Citak M, et al. (2015) Exoskeletons for rehabilitation of patients with spinal cord injuries: Options and limitations. Unfallchirurg 118: 130-137.

15. van Hedel HJ, Dietz V; European Multicenter Study on Human Spinal Cord Injury (EM-SCl) Study Group (2009) Walking during daily life can be validly and responsively assessed in subjects with a spinal cord injury. Neurorehabil Neural Repair 23: 117-124.

16. Rossier P, Wade DT (2001) Validity and reliability comparison of 4 mobility measures in patients presenting with neurologic impairment. Arch Phys Med Rehabil 82: 9-13.

17. van Hedel HJ, Wirz M, Dietz V (2008) Standardized assessment of walking capacity after spinal cord injury: The European network approach. Neurol Res 30: $61-73$.

18. Dittuno PL, Ditunno JF, Dittuno JF (2001) Walking index for spinal cord injury (WISCI II): Scale revision. Spinal Cord 39: 654-656.

19. Kim MO, Burns AS, Ditunno JF Jr, Marino RJ (2007) The assessment of walking capacity using the walking index for spinal cord injury: Self-selected versus maximal levels. Arch Phys Med Rehabil 88: 762-767.

20. Winchester P, McColl R, Querry R, Foreman N, Mosby J, et al. (2005) Changes in supraspinal activation patterns following robotic locomotor therapy in motorincomplete spinal cord injury. Neurorehabil Neural Repair 19: 313-324.

21. Aach M, Cruciger O, Sczesny-Kaiser M, Höffken O, Meindl RC, et al. (2014) Voluntary driven exoskeleton as a new tool for rehabilitation in chronic spinal cord injury: A pilot study. Spine J 14: 2847-2853.

22. Sczesny-Kaiser $M$, Kowalewski $R$, Schildhauer TA, Aach $M$, Jansen $O$, et al. (2017) Treadmill training with HAL exoskeleton-A novel approach for symptomatic therapy in patients with limb-girdle muscular dystrophy-preliminary study. Front Neurosci 11: 449.

23. Cruciger O, Schildhauer TA, MeindI RC, Tegenthoff M, Schwenkreis $P$, et al. (2016) Impact of locomotion training with a neurologic controlled hybrid assistive limb (HAL) exoskeleton on neuropathic pain and health related quality of life (HRQoL) in chronic SCl: A case study. Disabil Rehabil Assist Technol 11: 529-534. 